\section{Performance evaluation of a rectifier column using gamma column scanning}

Denis D. Aquino, Janice P. Mallillin, Ramoncito F. Sulit, Frederick C. Hila, Ivy Angelica A. Nuñez, Adelina D. M. Bulos

Abstract. Rectifier columns are considered to be a critical component in petroleum refineries and petrochemical processing installations as they are able to affect the overall performance of these facilities. It is deemed necessary to monitor the operational conditions of such vessels to optimize processes and prevent anomalies which could pose undesired consequences on product quality that might lead to huge financial losses. A rectifier column was subjected to gamma scanning using a $10-\mathrm{mCi}$ Co-60 source and a 2 -inch-long detector in tandem. Several scans were performed to gather information on the operating conditions of the column under different sets of operating parameters. The scan profiles revealed unexpected decreases in the radiation intensity at vapour levels between trays 2 and 3, and between trays 4 and 5. Flooding also occurred during several scans which could be attributed to parametric settings.

Keywords: gamma column scanning $\bullet$ distillation column $\bullet$ density profile $\bullet$ processing plant $\bullet$ refinery

D. D. Aquino ${ }^{\bowtie}$, J. P. Mallillin, R. F. Sulit, F. C. Hila,

I. A. A. Nuñez, A. D. M. Bulos

Philippine Nuclear Research Institute,

Commonwealth Ave., Diliman,

Quezon City, 1101 Metro Manila, Philippines,

Tel.: +632929 6011, Fax: +632920 1646,

E-mail: ddaquino@pnri.dost.gov.ph

Received: 28 March 2017

Accepted: 4 December 2017

\section{Introduction}

One of the critical components of petroleum refineries and petrochemical processing plants are distillation columns. The functionality of these columns according to their intended design could affect the overall performance of these kinds of installations. A kind of distillation column or column in petroleum refineries are rectifier or rectifying columns.

A rectifying column uses counter-current distillation to remove less volatile components from a liquid phase [1]. The process starts by introducing the liquid mixture (feed) to be separated at the bottom of the column which is brought to its boiling point. The vapour produced moves upwards inside the column and is released at the top to be condensed. A portion of the distillate is re-introduced inside the column as reflux liquid which moves downwards in the opposite direction to the movement of the vapour phase. The concentration of volatile components in the distillate that exits the column and the process is higher.

The nature of the process that takes place inside a rectifier column entails constant monitoring of process and output parameters for irregularities and abnormalities. Such processes pose severe consequences to the operation of the plant and quality of the product that could lead to substantial financial losses. The monitoring and inspection of 
the operational conditions of these columns is thus paramount.

A widely known technique for the online investigation of distillation columns in petroleum and chemical processing plants is the gamma column scanning technique. It incorporates the use of a sealed radioactive material to determine the operating conditions of distillation columns or process vessels. The technique is able to determine a number of process anomalies and provide information concerning the optimization of column performance as well as identify requirements for maintenance operations [2]. Some of these malfunctions that the gamma column scanning technique can identify are mechanical in nature such as displaced or missing trays, or related to problems that address processes and rates, e.g. entrainment, flooding and maldistribution.

The results of gamma scanning performed on a rectifier column which was exhibiting observable intermittent anomalies are reported in this paper. The objective of scanning is to gather information for the operators and process engineers to determine the condition of the vessel under variable process parameters prior to arranging the necessary maintenance operations. The investigation was conducted without shutting down the process so as not to disrupt the operation that would entail costly losses in terms of production.

\section{Methodology}

Gamma column scanning utilizes a radioactive source and a detector for gathering information on the condition of a column. The transmitted radiation intensity that passed through the column, measured in counts per seconds by a detector on the opposite side in the same horizontal plane, is plotted against the density profile of the column. The conversion of radiation intensity in terms of density is based on the equation $I=I_{o} e^{-\mu \rho x}$ which describes the fundamental relationship between the transmitted intensity $I$ and the initial intensity $I_{o}$ that traversed through a thickness $x$ of material with density $\rho$ and mass absorption coefficient $\mu[3,4]$.

The resulting density profile of a distillation column can provide a wealth of information that could reveal mechanical- and process-related conditions of the vessel [2]. This data could help plant engineers and operators to make the necessary plans and arrangements to optimize the performance of a column, extend run-times and reduce periods of shut down for routine maintenance.

The column under investigation is $1 \mathrm{~m}$ in diameter and has a wall thickness of $10 \mathrm{~mm}$. A maximum height of approximately $440 \mathrm{~cm}$ along the column was scanned for this activity. A NaI scintillation detector was used to measure the transmitted radiation from a $10 \mathrm{mCi}$ collimated $\mathrm{Co}-60$ radioactive source. The measured radiation intensity was automatically logged on a computer through a rate meter. Figure 1 shows the set-up for scanning and the equipment used in this activity. Scanning was done in such a way that

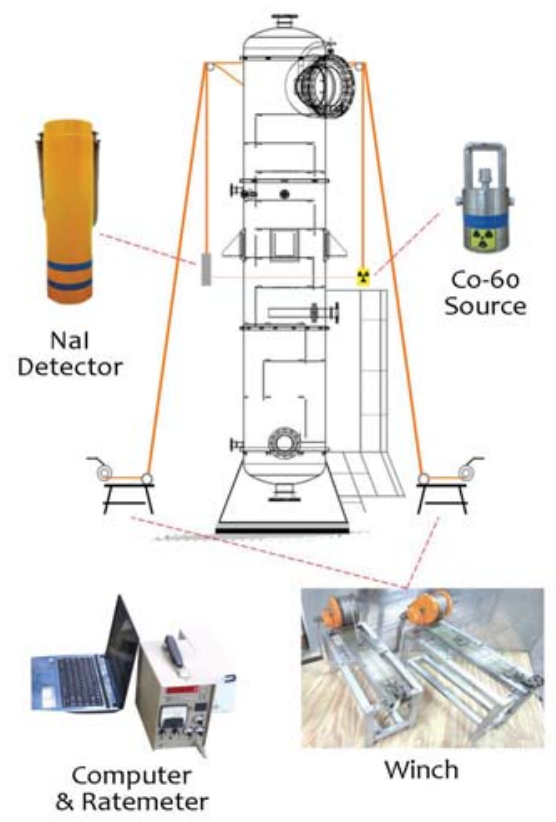

Fig. 1. Column scanning set-up and equipment used.

the downcomers were avoided in order to prevent ambiguity in the interpretation of results. The scanning orientation is presented in Fig. 2.

A total of twelve (12) scans were performed to gather information on the condition of the column under different operating parameters. Each scan represents a different set of operating parameters determined by the plant engineers and operators. In this activity, the operating parameters were not known by or revealed to the authors. Variations in the process parameters were made by operators who gave instructions to commence scanning upon completion of changes to parameter settings.

\section{Results and discussion}

The resulting density profiles were plotted and compared with the engineering diagram as shown in Fig. 3. The $x$ axis pertains to the radiation intensity, in counts, along the traversed length of the gamma

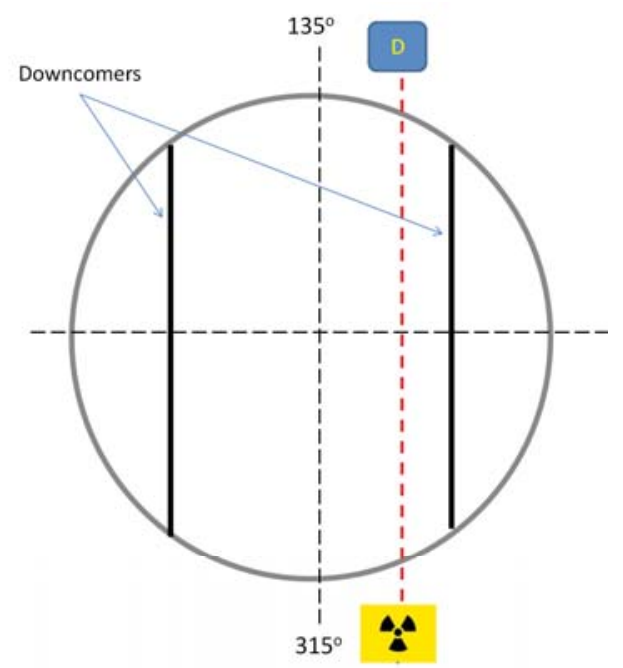

Fig. 2. Scanning orientation. 




Fig. 3. Resulting density profiles for each scan.

rays while the $y$ axis is the corresponding elevation with regard to the height of the column.

Scan 1 reflects the presence of all trays at their respective positions. Interestingly, the intensities dropped at the vapour level between trays 2 and 3, and between trays 4 and 5 . Radiation intensities at these levels are expected to be high since there are no obstructions along the pathway of the gamma rays. These same observations were also present in scans $2,4-7$ and 9-11. The low radiation intensities that were detected may be due to a high liquid level due to weeping, foaming or a blockage. Low radiation intensity readings were also recorded just below tray 1 in these scan lines which could indicate a high liquid level at the bottom of the column or a high density material below the tray obstructing the gamma rays along the pathway.

Flooding conditions were observed during scans 3, 6, 8 and 12. During scan 3, flooding started in tray 1 and reached up to tray 7 . Flooding during scan 6 reached tray 1 , while during scan 8 , it reached tray 5 . During scan 12, flooding rose up to tray 2. The differences in the height of the tray up to which flooding occurred could be attributed to the varying feed-rate settings during the scans.

\section{Conclusion}

The gamma column scanning technique was used to determine the operating conditions of a rectifier under various process parameter settings. Twelve scan lines were plotted to represent the conditions inside the column under twelve parameter settings set by the operators. Scan lines 1-2, 4-7 and 9-11 indicate unexpected low radiation intensities at the vapour level between trays 2 and 3, and between trays 4 and 5 . This could indicate a high liquid level due to foaming, weeping or a blockage.

The technique was also able to identify the occurrence of flooding under certain parameter settings as reflected in scan lines 3, 6, 8 and 12 . This could possibly be attributed to the parameter settings such as the different feed rates as well as the presence of a blockage along the pathway of the liquid flow.

Acknowledgment. The authors acknowledge the support of the Philippine Council for Industry, Energy and Emerging Technology Research and Development - Department of Science and Technology (PCIEETRD-DOST) for their support in funding this capacity development project of the Philippine Nuclear Research Institute (PNRI) concerning gamma scanning. We are grateful for the assistance provided by the International Atomic Energy Agency (IAEA) with regard to the capacity development initiatives of PNRI. A special mention is given to Mr. Patrick Brisset, Technical Officer of IAEA and Mr. Dhanaj Saengchantr of the Thailand Institute of Nuclear Technology (TINT) for their assistance and consultancy with regard to our projects and activities.

\section{References}

1. Brown, G. G. (1950). Unit operations. New York: Wiley.

2. Jaafar, A. (2005). Gamma ray scanning for troubleshooting, optimization and predictive maintenance of distillation columns. Hydrocarbon Asia, Jan/Feb, 62-65.

3. Charlton, J. S. (1985). Radioisotope techniques for problem-solving in industrial process plants. Glasgow: Leonard Hill.

4. Kim, J., Jung, S., \& Kim, J. (2007). The identification of flooding phenomenon in a column of refinery process by gamma absorption technique. J. Label. Compd. Radiopharm., 50(5/6), 605-606. doi: 10.1002/jlcr.1299. 